\title{
Resistência de clones de Eucalyptus ao psilídeo-de-concha
}

\author{
Joelma Melissa Malherbe Camargo ${ }^{1}$, Keti Maria Rocha Zanol ${ }^{1}$, Dalva Luiz de Queiroz ${ }^{2}$, Renato Antonio Dedececk ${ }^{2}$, Edilson Batista \\ Oliveira $^{2}$, Raul Cesar Nogueira Melido ${ }^{3}$
}

\author{
${ }^{1}$ Universidade Federal do Paraná, Departamento de Zoologia, CP 19.020, CEP 81531-990, Curitiba, PR, Brasil \\ ${ }^{2}$ Embrapa Florestas, Estrada da Ribeira, Km 111, CP 319, CEP 83411-000, Colombo, PR, Brasil \\ ${ }^{3}$ Votorantim Siderurgia, Fazenda Bom Sucesso, s/nº, CP 04, CEP 38780-000, João Pinheiro, MG, Brasil
}

\author{
"Autor correspondente: \\ dalva.queiroz@embrapa.br \\ Termos para indexação: \\ Eucalipto \\ Entomologia florestal \\ Glycaspis brimblecombei \\ Index terms: \\ Eucalyptus \\ Forest entomology \\ Glycaspis brimblecombei
}

Histórico do artigo:

Recebido em 27/03/2013

Aprovado em 25/03/2014

Publicado em 04/04/2014

doi: 10.4336/2014.pfb.34.77.504

\begin{abstract}
Resumo - O objetivo deste trabalho foi avaliar a resistência de clones comerciais de $E$. camaldulensis e E. urophylla ao ataque de Glycaspis brimblecombei Moore (Hemiptera: Aphalaridae). O experimento foi conduzido entre maio de 2007 e abril de 2008, em João Pinheiro, MG, com cinco clones de E. camaldulensis e cinco de E. urophylla. O delineamento experimental foi de blocos ao acaso, contendo 25 plantas por parcela, com quatro repetições. Mensalmente foram coletadas quatro folhas das nove plantas centrais de cada parcela, quantificando o número de ovos e ninfas do inseto. As maiores médias para ovos e ninfas foram observadas para os clones de E. camaldulensis, os quais foram classificados como altamente suscetíveis ao ataque do psilídeo-de-concha. Os clones de E. urophylla apresentaram menores médias para ovos e ninfas, sendo classificados como resistentes.
\end{abstract}

\section{Resistence of Eucalyptus clones to the red gum lerp psyllid}

\begin{abstract}
The objective of this work was to evaluate the different resistance of commercial E. camaldulensis and E. urophylla clones to the attack of (Glycaspis brimblecombei) Moore (Hemiptera: Aphalaridae). The experiment was carried out between May of 2007 and April of 2008, in João Pinheiro, Minas Gerais State, Brazil, with 5 clones of $E$. camaldulensis and 5 of E. urophylla. The experimental design was randomized blocks, with 25 plants per plot, and four replications. Four leaves of nine plants were collected in the center of each plot to quantify the number of eggs and nymphs. The highest averages for eggs and nymphs were observed in E. camaldulensis clones, which were classified as highly susceptible to the red-gum-lerp-psyllid attack. Clones of E. urophylla had lower averages for eggs and nymphs, and it was classified as resistant.
\end{abstract}

\section{Introdução}

O Brasil detém a maior área plantada de eucalipto do mundo, destinada principalmente à produção de celulose e papel, chapas de fibras e carvão vegetal, que abastece as indústrias de base florestal (Brasil, 2010). Em 2012, a área total ocupada por plantações florestais no Brasil chegou a 7,18 milhões de hectares. Deste total, as áreas plantadas com eucalipto ocuparam $70,8 \%$, seguida pelo pinus (22\%) e as demais espécies ( $7,2 \%)$. Os plantios de eucalipto e pinus estão concentrados em Minas Gerais, São Paulo, Paraná, Santa Catarina, Bahia e Mato Grosso do Sul. (Florestas..., 2013).

A eucaliptocultura é intensiva e baseada principalmente em florestas clonais, formadas com materiais-elite de elevada produtividade média, chegando a atingir valores 
da ordem de $60 \mathrm{~m}^{3} \mathrm{ha}^{-1}$ ano $^{-1}$ (Mora \& Garcia, 2000). Em geral, a seleção de clones baseia-se na produtividade e qualidade da floresta, levando-se em consideração a suscetibilidade de plantas ao ataque de patógenos e insetos. Desde a década de 1980, vários estudos têm sido conduzidos, visando conhecer a suscetibilidade ou resistência das espécies de Eucalyptus a insetospraga, tais como lagartas (Zanuncio, 1993), besouros desfolhadores (Anjos \& Majer, 2003), formigas cortadeiras (Santana \& Anjos 1989; Santana \& Couto 1990) e psilídeos (Brennan \& Weinbaum, 2001a, 2001b; Hidalgo Reys, 2005; Santana et al. 2010; Pereira 2013).

A busca por plantas resistentes ou tolerantes ao ataque de insetos tem sido uma alternativa economicamente viável para conter as populações das pragas em níveis inferiores ao de dano econômico, sem causar prejuízos ao ambiente e sem ônus ao produtor (Queiroz et al., 2012).

Com o aumento da área plantada com Eucalyptus, principalmente para produção de energia e celulose, os problemas relacionados às pragas têm se agravado. O psilídeo-de-concha, Glycaspis brimblecombei (Moore, 1964) (Hemiptera: Psylloideae), recentemente reclassificado dentro da família Aphalaridae (Burckhardt \& Ouvrard, 2012), é nativo da Austrália, mas encontrase distribuído por vários continentes, como África, América e Europa (Brennan et al. 1999; Bouvet et al. 2005; Burckhardt et al 2008; Peris-Felipo et al. 2011). No Brasil, está presente em diversas regiões (Wilcken et al., 2003, Santana et al. 2003b; Santana \& Burckhardt, 2007; Breda et al. 2010; Burckhardt \& Queiroz, 2012), com potencial de continuar se dispersando para as novas fronteiras florestais (Queiroz et al. 2013). Os danos causados pelo inseto são intensos, com desfolha de $20 \%$ a $30 \%$, secamento dos ponteiros, indução de fumagina e mortalidade de árvores (Wilcken et al., 2003).

Dependendo das condições climáticas, os psilídeos tendem a ser univoltinos ou bivoltinos (regiões temperadas) ou polivoltinos, com sobreposição de gerações nos trópicos, com várias gerações por ano (Burckhardt, 1994; Hollis, 2004; Santana et al., 2010). Hodkinson (2009) analisou as características do ciclo de vida de 342 espécies de psilídeos de todo o mundo, concluindo que, na maioria dos casos, a temperatura e disponibilidade de água atuam diretamente no inseto ou via hospedeiro, determinando seus ciclos de vida. A biologia dos psilídeos é, portanto, sincronizada com a fenologia de seus hospedeiros. As populações dos psilídeos podem ser afetadas e/ou controladas por uma série de inimigos naturais, mas em florestas plantadas isto se torna menos eficiente e os ataques são mais frequentes. $\mathrm{Na}$ Austrália, região de origem do eucalipto e também do psilídeo-de-concha, e adicionalmente no Brasil, em períodos mais frios e secos, as plantas de eucalipto se encontram mais estressadas e, consequentemente, concentram nitrogênio, o que favorece o aumento populacional dos psilídeos (White, 1969; Santana et al, 2003a).

$\mathrm{O}$ ataque do psilídeo-de-concha não é homogêneo entre espécies de Eucalyptus, sendo consistentemente mais intenso em E. camaldulensis e E. tereticornis (Paine et al., 2000; Brennan et al., 2001). Em sua região de origem, o principal hospedeiro de G. brimblecombei é E. camaldulensis, uma das espécies de eucalipto com maior distribuição na Austrália, abrangendo as regiões temperadas, bem como áreas secas, ocorrendo principalmente ao longo do curso de rios (Moore 1975; Queiroz et al. 2013). Assim como o E. camaldulensis, o gênero Glycaspis também possui ampla distribuição geográfica na sua região de origem, mas segundo Moore (1975), as espécies de Glycaspis na Austrália são diferentemente influenciadas pela temperatura, precipitação, presença do hospedeiro e até mesmo pelo tipo de solo. Além de E. camaldulensis, $G$. brimblecombei desenvolve-se em outras espécies de Eucalyptus, tais como: E. tereticornis, E. blakelyi, E. brasssiana, E. bridgesiana, E. camphora, E. dealbata, E. diversicolor, E. globulus, E. mannifera, E. mannifera maculosa, E. nitens e E. sideroxylon (Brennan et al., 2001; Hollis, 2004).

No Brasil, o psilídeo-de-concha já foi observado nas espécies E. camaldulensis, E. tereticornis, E. urophylla, E. grandis, E. globulus, E. viminalis, E. paniculata, E. robusta, E. saligna bem como em clones híbridos de $E$. grandis $x$ E. urophylla ("Urograndis") (Wilcken et al., 2003; Firmino-Winckler et al., 2009).

Vários autores têm observado que o E.camaldulensis é a espécie mais susceptível ao psilídeo-de-concha (Hidalgo Reys, 2005; Pereira et al., 2013). No entanto, existem variações entre as variedades, procedências e clones. Apesar do E. camaldulensis ser o mais atacado pelo psilídeo-de-concha, esta é uma das espécies comerciais com maior resistência ao déficit hídrico, portanto, a mais plantada nas regiões secas, como o norte de Minas Gerais e noroeste da Bahia. 
O objetivo deste estudo foi avaliar os clones comerciais de $E$. camaldulensis $(7,58,62,10$ e 6$)$, e de E. urophylla (19, 36, 2, I224 e I042), visando identificar os mais resistentes ao psilídeo-de-concha e indicar o melhor material para utilização em larga escala em futuros plantios.

\section{Material e métodos}

O estudo foi conduzido em uma área de plantio comercial de eucalipto da empresa Votorantim Siderurgia (Fazenda Santa Cecília), localizada no Município de João Pinheiro, região noroeste do Estado de Minas Gerais. A Fazenda encontra-se a $450 \mathrm{~km}$ da cidade de Belo Horizonte, MG, com área total de 26.000 hectares. O clima é do tipo tropical úmido, subúmido, com precipitação média anual de $1.300 \mathrm{~mm}$ e temperatura média anual de $20,5{ }^{\circ} \mathrm{C}$, apresentando déficit hídrico de 60 a $120 \mathrm{~mm}$, entre os meses de maio e agosto. Os solos são classificados como Luvissolos férricos, Ferralsolos ácricos e Arenossolos ferrálicos, com relevo plano. A altitude local varia de 600 a $1.000 \mathrm{~m}$ (Mellido Filho, 1994). Esta área foi selecionada por haver plantios de eucalipto com uma alta infestação do psilídeo-deconcha, com surtos nos anos anteriores à instalação do experimento.

Para os testes, foram escolhidos os clones comerciais mais utilizados na região. As mudas dos clones de $E$. camaldulensis (CL 6, CL 10, CL 7, CL 58 e CL 62) e $E$. urophylla (CL 2, CL 19, CL I0 42, CL I224 e CL 36) foram produzidas no mês de janeiro de 2007, por meio de miniestaquia em viveiro comercial e, posteriormente, rustificadas a pleno sol.

O plantio em campo foi realizado no mês de maio de 2007, com as mudas apresentando aproximadamente 120 dias. Foram plantadas no espaçamento de $3 \times 3 \mathrm{~m}$, totalizando 1.111 plantas por hectare.

Foram coletadas mensalmente folhas dos dez clones, por um período de um ano, entre maio de 2007 a abril de 2008, sendo avaliadas as nove plantas centrais de cada parcela, coletando-se quatro folhas por planta. A coleta das folhas foi manual, no terço médio da planta, nas direções norte, sul, leste e oeste. Imediatamente após a coleta as folhas foram acondicionadas em sacos de papel e encaminhadas ao laboratório de Entomologia da Embrapa Florestas, onde foram examinadas sob microscópio estereoscópico Olympus SZ 40, em um aumento entre 40 a 100 vezes.
O delineamento experimental utilizado foi de blocos ao acaso, contendo 25 plantas por parcela, com quatro repetições. Os dados obtidos foram submetidos à análise de variância (ANOVA), onde se empregou o teste de correlação e teste Tukey a 5\% de significância.

\section{Resultados e discussão}

Foram analisadas cento e quarenta e quatro folhas por tratamento, perfazendo um total de mil quatrocentos e quarenta folhas por avaliação, onde os ovos e ninfas de G. brimblecombei foram quantificados.

Nos meses de pouca ou nenhuma chuva na região (entre maio e agosto) houve um aumento populacional da praga, com o pico de ovos em junho de 2007 (Tabela 1) e de ninfas no mês seguinte (Tabela 2). Estes resultados são compatíveis com os observados por Ferreira et al. (2009), no Município de Garça, SP, onde o maior índice de infestação foi encontrado em agosto, mês de menor precipitação.

Analisando-se cada mês separadamente (Tabela 1), observam-se diferenças significativas entre os clones de E. camaldulensis, como por exemplo no mês $06 / 2007$, onde os clones 10 e 7 apresentaram mais ovos que os demais. Porém, estas diferenças não são consistentes, ou seja, variam mês a mês.

A ocorrência de ninfas também variou de acordo com os clones e data de coleta (Tabela 2). Neste caso, apenas o clone 6 se diferenciou dos demais clones de E. camaldulensis.

Como estes índices (ovos e ninfas) variaram inconsistentemente durante o período avaliado, optou-se por analisar a resistência e susceptibilidade do eucalipto ao psilídeo baseada na média do período (Tabela 3 ). De acordo com os dados médios, todos os clones de E. camaldulensis avaliados apresentaram as maiores médias de números de ovos, do psilídeo-de-concha do que os de E. urophylla, evidenciando maior preferência do psilídio-de-concha por ovipositar em híbridos com esta espécie.

Quanto ao número médio de ninfas, obteve-se resultado semelhante, ou seja, o maior número de ninfas ocorreu para os clones de E. camaldulensis, diferindo estatisticamente dos clones de E. urophylla (Tabela $3)$. Estes resultados corroboram trabalhos anteriores (Brennan et al., 2001; Pereira et al., 2013), onde os autores observaram que o E. camaldulensis é o mais susceptível. 
Tabela 1. Número médio de ovos do psilídeo-de-concha Glycaspis brimblecombei em folhas de clones de Eucalyptus spp. no período de maio/2007 a abril/2008, em João Pinheiro, MG.

\begin{tabular}{|c|c|c|c|c|c|c|c|c|c|c|c|c|}
\hline \multirow[b]{2}{*}{ Clones } & \multicolumn{12}{|c|}{ Número médio de ovos } \\
\hline & 05/07 & 06/07 & 07/07 & 08/07 & 09/07 & $10 / 07$ & 11/07 & $12 / 07$ & $01 / 08$ & $02 / 08$ & $03 / 08$ & $04 / 08$ \\
\hline \multicolumn{13}{|c|}{ E. camaldulensis } \\
\hline clone 6 & $0,31 \mathrm{a}$ & $6,55 b$ & $4,67 b$ & $3,55 \mathrm{a}$ & $0,80 \mathrm{a}$ & $0,45 \mathrm{a}$ & $0 \mathrm{a}$ & $0 \mathrm{a}$ & $0 \mathrm{a}$ & $0 \mathrm{a}$ & $0 \mathrm{a}$ & $0 \mathrm{a}$ \\
\hline clone 10 & $0,37 \mathrm{a}$ & $11,43 a$ & $3,52 b$ & $4,18 \mathrm{a}$ & $0,58 \mathrm{a}$ & $0,10 \mathrm{~b}$ & $0 \mathrm{a}$ & $0 \mathrm{a}$ & $0 \mathrm{a}$ & $0 \mathrm{a}$ & $0 \mathrm{a}$ & $0 \mathrm{a}$ \\
\hline clone 7 & $0,38 \mathrm{a}$ & $12,06 \mathrm{a}$ & $8,75 \mathrm{a}$ & $2,40 \mathrm{~b}$ & $0,32 b$ & $0,11 b$ & $0 \mathrm{a}$ & $0 \mathrm{a}$ & $0 \mathrm{a}$ & $0 \mathrm{a}$ & $0 \mathrm{a}$ & $0 \mathrm{a}$ \\
\hline clone 58 & $0,08 b$ & $8,97 \mathrm{~b}$ & $4,58 b$ & $3,00 \mathrm{~b}$ & $0 \mathrm{c}$ & $0,48 \mathrm{a}$ & $0,02 \mathrm{a}$ & $0 \mathrm{a}$ & $0 \mathrm{a}$ & $0 \mathrm{a}$ & $0 \mathrm{a}$ & $0 \mathrm{a}$ \\
\hline clone 62 & $0,38 \mathrm{a}$ & $7,38 b$ & $4,16 b$ & $3,72 \mathrm{a}$ & $0 \mathrm{c}$ & $0,07 \mathrm{~b}$ & $0 \mathrm{a}$ & $0 \mathrm{a}$ & $0,05 \mathrm{a}$ & $0 \mathrm{a}$ & $0 \mathrm{a}$ & $0 \mathrm{a}$ \\
\hline \multicolumn{13}{|l|}{ E. urophylla } \\
\hline clone 2 & $0,21 \mathrm{a}$ & $0,80 \mathrm{c}$ & $0,11 d$ & $0,08 \mathrm{~d}$ & $0,02 \mathrm{c}$ & $0,13 b$ & $0 \mathrm{a}$ & $0 \mathrm{a}$ & $0 \mathrm{a}$ & $0 \mathrm{a}$ & $0 \mathrm{a}$ & $0 \mathrm{a}$ \\
\hline clone 19 & $0,31 \mathrm{a}$ & $2,36 \mathrm{c}$ & $0,31 \mathrm{c}$ & $0,23 \mathrm{c}$ & $0,02 \mathrm{c}$ & $0 \mathrm{c}$ & $0 \mathrm{a}$ & $0 \mathrm{a}$ & $0 \mathrm{a}$ & $0 \mathrm{a}$ & $0 \mathrm{a}$ & $0 \mathrm{a}$ \\
\hline clone I042 & $0 \mathrm{~b}$ & $0,20 \mathrm{c}$ & $0,08 \mathrm{~d}$ & $0,12 d$ & $0,02 \mathrm{c}$ & $0,01 \mathrm{c}$ & $0 \mathrm{a}$ & $0 \mathrm{a}$ & $0 \mathrm{a}$ & $0 \mathrm{a}$ & $0 \mathrm{a}$ & $0 \mathrm{a}$ \\
\hline clone I224 & $0,02 \mathrm{a}$ & $0,81 \mathrm{c}$ & $0,02 \mathrm{~d}$ & $0,43 \mathrm{c}$ & $0,20 \mathrm{~b}$ & $0,40 \mathrm{a}$ & $0 \mathrm{a}$ & $0 \mathrm{a}$ & $0 \mathrm{a}$ & $0 \mathrm{a}$ & $0 \mathrm{a}$ & $0 \mathrm{a}$ \\
\hline clone 36 & $0,01 \mathrm{~b}$ & $0,95 \mathrm{c}$ & $0,58 \mathrm{c}$ & $0,12 \mathrm{~d}$ & $0,25 b$ & $0 \mathrm{c}$ & $0 \mathrm{a}$ & $0 \mathrm{a}$ & $0 \mathrm{a}$ & $0 \mathrm{a}$ & $0 \mathrm{a}$ & $0 \mathrm{a}$ \\
\hline
\end{tabular}

*Médias seguidas pela mesma letra nas colunas não diferem significativamente entre si pelo teste Tukey $(\mathrm{p}=0,05)$.

Tabela 2. Número médio de ninfas do psilídeo-de-concha Glycaspis brimblecombei folhas de clones de Eucalyptus spp. no período de maio/2007 a abril/2008, em João Pinheiro, MG.

\begin{tabular}{|c|c|c|c|c|c|c|c|c|c|c|c|c|}
\hline \multirow[b]{2}{*}{ Clones/data } & \multicolumn{12}{|c|}{ Número médio de ninfas } \\
\hline & 05/07 & $06 / 07$ & 07/07 & $\mathbf{0 8} / 07$ & $09 / 07$ & 10/07 & 11/07 & $12 / 07$ & $01 / 08$ & $02 / 08$ & $03 / 08$ & $04 / 08$ \\
\hline \multicolumn{13}{|c|}{ E. camaldulensis } \\
\hline clone 6 & $0,03 \mathrm{a}$ & $0,25 \mathrm{a}$ & $5,36 \mathrm{a}$ & $1,31 \mathrm{a}$ & $1,35 \mathrm{a}$ & $0,37 \mathrm{a}$ & $0 \mathrm{a}$ & $0 \mathrm{a}$ & $0 \mathrm{a}$ & $0 \mathrm{a}$ & $0 \mathrm{a}$ & $0 \mathrm{a}$ \\
\hline clone 10 & $0 \mathrm{a}$ & $0,06 b$ & $5,82 \mathrm{a}$ & $0,82 \mathrm{a}$ & $1,20 \mathrm{a}$ & $0,01 b$ & $0 \mathrm{a}$ & $0 \mathrm{a}$ & $0 \mathrm{a}$ & $0 \mathrm{a}$ & $0 \mathrm{a}$ & $0 \mathrm{a}$ \\
\hline clone 7 & $0 \mathrm{a}$ & $0,08 b$ & $8,37 \mathrm{a}$ & $1,30 \mathrm{a}$ & $0,97 \mathrm{a}$ & $0,02 b$ & $0 \mathrm{a}$ & $0 \mathrm{a}$ & $0 \mathrm{a}$ & $0 \mathrm{a}$ & $0 \mathrm{a}$ & $0 \mathrm{a}$ \\
\hline clone 58 & $0 \mathrm{a}$ & $0,08 b$ & $7,53 \mathrm{a}$ & $1,13 \mathrm{a}$ & $0,78 \mathrm{a}$ & $0,15 \mathrm{a}$ & $0,02 \mathrm{a}$ & $0 \mathrm{a}$ & $0 \mathrm{a}$ & $0 \mathrm{a}$ & $0 \mathrm{a}$ & $0 \mathrm{a}$ \\
\hline clone 62 & $0,01 \mathrm{a}$ & $0,11 b$ & $6,91 \mathrm{a}$ & $1,33 \mathrm{a}$ & $0,26 b$ & $0,01 b$ & $0 \mathrm{a}$ & $0 \mathrm{a}$ & $0,05 \mathrm{a}$ & $0 \mathrm{a}$ & $0 \mathrm{a}$ & $0 \mathrm{a}$ \\
\hline \multicolumn{13}{|l|}{ E. urophylla } \\
\hline clone 2 & $0,01 \mathrm{a}$ & $0,01 \mathrm{c}$ & $0,51 \mathrm{c}$ & $0,02 b$ & $0,04 \mathrm{c}$ & $0,02 b$ & $0 \mathrm{a}$ & $0 \mathrm{a}$ & $0 \mathrm{a}$ & $0 \mathrm{a}$ & $0 \mathrm{a}$ & $0 \mathrm{a}$ \\
\hline clone 19 & $0,01 \mathrm{a}$ & $0,03 \mathrm{c}$ & $1,40 \mathrm{~b}$ & $0,02 b$ & $0,18 b$ & $0 \mathrm{~b}$ & $0 \mathrm{a}$ & $0 \mathrm{a}$ & $0 \mathrm{a}$ & $0 \mathrm{a}$ & $0 \mathrm{a}$ & $0 \mathrm{a}$ \\
\hline clone I042 & $0 \mathrm{a}$ & $0,01 \mathrm{c}$ & $0,16 \mathrm{c}$ & $0,03 b$ & $0,18 b$ & $0 \mathrm{~b}$ & $0 \mathrm{a}$ & $0 \mathrm{a}$ & $0 \mathrm{a}$ & $0 \mathrm{a}$ & $0 \mathrm{a}$ & $0 \mathrm{a}$ \\
\hline clone I224 & $0,03 \mathrm{a}$ & $0 \mathrm{c}$ & $0,22 \mathrm{c}$ & $0,11 \mathrm{~b}$ & $0,15 b$ & $0,02 b$ & $0 \mathrm{a}$ & $0 \mathrm{a}$ & $0 \mathrm{a}$ & $0 \mathrm{a}$ & $0 \mathrm{a}$ & $0 \mathrm{a}$ \\
\hline clone 36 & $0 \mathrm{a}$ & $0,03 \mathrm{c}$ & $1,00 \mathrm{~b}$ & $0,13 b$ & $0,03 \mathrm{c}$ & $0 \mathrm{~b}$ & $0 \mathrm{a}$ & $0 \mathrm{a}$ & $0 \mathrm{a}$ & $0 \mathrm{a}$ & $0 \mathrm{a}$ & $0 \mathrm{a}$ \\
\hline
\end{tabular}

*Médias seguidas pela mesma letra nas colunas não diferem significativamente entre si pelo teste Tukey $(\mathrm{p}=0,05)$.

O ajuste de modelos lineares com coeficiente angular positivo entre o número médio de ovos obtido em junho e o de ninfas em julho, bem como para o número médio de ovos em julho e o de ninfas em agosto (Figura 1) demonstram que o número de ninfas de um mês é diretamente proporcional ao número de ovos no mês anterior. Assim, é possível estimar o número de ninfas a partir da amostragem do número de ovos, utilizando essa estimativa como ferramenta para o manejo do psilídeode-concha e planejamento de controle.
Considerando-se o número médio de ovos e de ninfas observados nas folhas do terço médio da copa dos clones avaliados, os cinco clones de E. camaldulensis foram classificados como altamente susceptíveis e os outros cinco clones de E. urophylla, como resistentes (Tabela 3). Apesar do psilídeo-de-concha se alimentar de várias espécies de eucalipto, ele prefere colonizar espécies do subgênero Symphyomyrtus, seção Exsertaria (Pryor \& Johnson, 1971), particularmente E. camaldulensis (Paine et al., 2000). 
Tabela 3. Número médio de ovos e ninfas do psilídeo-deconcha Glycaspis brimblecombei em folhas de clones de Eucalyptus spp., coletadas no pico populacional de ovos e ninfas, no período de $05 / 07$ a $02 / 08$ na empresa Votorantim Siderurgia, João Pinheiro, MG.

\begin{tabular}{|c|c|c|c|c|}
\hline \multirow{2}{*}{ Espécie } & \multirow{2}{*}{ Clone } & Ovos & Ninfas & \multirow{2}{*}{$\begin{array}{l}\text { Grau de } \\
\text { resistência }\end{array}$} \\
\hline & & \multicolumn{2}{|c|}{ número } & \\
\hline \multirow{5}{*}{ E. camaldulensis } & 7 & $8,37 \mathrm{a}$ & $12,06 \mathrm{a}$ & Susceptível \\
\hline & 58 & $7,53 \mathrm{a}$ & $8,97 \mathrm{a}$ & Susceptível \\
\hline & 62 & $6,91 \mathrm{a}$ & $7,38 \mathrm{a}$ & Susceptível \\
\hline & 10 & $5,82 \mathrm{a}$ & $11,43 a$ & Susceptível \\
\hline & 6 & $5,36 \mathrm{a}$ & $6,55 a$ & Susceptível \\
\hline \multirow{5}{*}{ E. urophylla } & 19 & $1,40 \mathrm{~b}$ & $2,36 b$ & Resistente \\
\hline & 36 & $1,00 \mathrm{~b}$ & $0,95 b$ & Resistente \\
\hline & 2 & $0,51 b$ & $0,80 \mathrm{~b}$ & Resistente \\
\hline & I042 & $0,22 b$ & $0,20 \mathrm{~b}$ & Resistente \\
\hline & I224 & $0,22 b$ & $0,81 b$ & Resistente \\
\hline
\end{tabular}

*Médias seguidas pela mesma letra nas colunas não diferem significativamente entre si pelo teste Tukey $(\mathrm{p}=0,05)$.

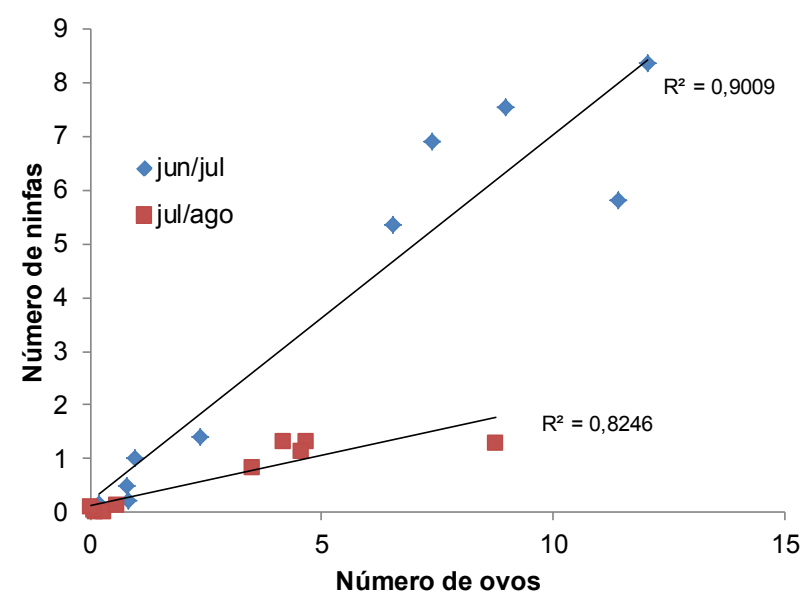

Figura 1. Análise de correlação entre o número médio de ovos e de ninfas, para o psilídeo-de-concha (Glycaspis brimblecombei) em folhas de diferentes clones de Eucalyptus camaldulensis e E. urophylla, no período de maior incidência da praga (junho a agosto), em João Pinheiro, MG.

Brennan et al. (2001) avaliaram a suscetibilidade de 21 espécies de Eucalyptus a G. brimblecombei, em São Francisco, Califórnia, EUA, com base na presença de ovos e de ninfas, e na ocorrência e gravidade de desfolha da árvore, sendo a maioria das espécies classificadas como resistentes ou tolerantes. Quatro espécies foram classificadas como moderadamente tolerantes ou suscetíveis, e apenas três espécies (E. camaldulensis E. rudis e E. tereticornis) apresentaram desfolha pesada e foram classificadas como moderadamente a altamente suscetíveis. Segundo os autores, as ceras epicuticulares das folhas parecem estar envolvidas com a resistência porque todas as espécies que possuem folhas do tipo "glaucous" (folhas esbranquiçadas, com grande quantidade de ceras) foram classificadas como resistentes.

Também baseado no número de ovos e ninfas, Wilcken et al. (2003) realizaram levantamento da infestação do psilídeo-de-concha em 22 espécies de eucalipto, classificando as espécies em resistentes e suscetíveis, sendo a maior infestação pela praga observada em $E$. camaldulensis.

Mesmo dentro da espécie E. camaldulensis, Glycaspis parece ter preferência por uma variedade, o que pode ser sugerido através da comparação da distribuição original de G. brimblecombei (Moore, 1975; Queiroz et al. 2013) com a distribuição natural de E. camaldulensis var. camaldulensis (Brooker, 2000; Centre for Plant Biodiversity Research, 2006). Estas diferenças na preferência do inseto para oviposição em diferentes variedades ou espécies (Brennan et al., 2001, Pereira et al., 2013)) também é refletida nos clones aqui avaliados (Tabela 3). Além de apresentarem uma boa resistência ao stress hídrico, os clones 58 e 62 (E. camaldulensis) são de grande importância para a região estudada, uma vez que possuem características físicas apropriadas para uso em postes, estacas, mourões e construção civil. No entanto, estes clones estão entre os mais atacados pelo psilídeo-de-conchas, o que demanda ações de manejo específicas para manter a praga sob controle.

Sugere-se que se identifique outros clones mais resistentes, para gradativamente substituir os atualmente em uso.

\section{Conclusões}

Todos os clones de E. camaldulensis foram classificados como susceptíveis ao psilídeo-de-concha G. brimblecombei.

Todos os clones de Eucalyptus urophylla foram classificados como resistentes ao psilídeo-de-concha G. brimblecombei.

A quantidade de ovos de G. brimblecombei em um mês pode ser eficiente para avaliar o número de ninfas do mês subsequente, facilitando o planejamento de controle. 


\section{Referências}

ANJOS, N. dos; MAJER, J. Leaf-eating beetles in Brazilian eucalypt plantations. Curtin University School of Environmental Biology, 2003. 33 p. (Bulletin, 23).

BOUVET, J. P. R.; HARRAND, L.; BURCKHARDT, D. Primera cita de Blastopsylla occidentalis y Glycaspis brimblecombei (Hemiptera: Psyllidae) para la República Argentina. Revista Sociedad Entomológica Argentina, Mendoza, v. 64, p. 99-102, 2005.

BRASIL. Ministério de Ciência e Tecnologia. Especial Projeto Genolyptus. Disponível em: <http:www.mct.gov.br/ genolyptus4htm>. Acesso em: 20 mar. 2010.

BREDA, M. O.; OLIVEIRA, J. V.; MOREIRA, A. N.; QUEIROZ, D. L. Registro de Glycaspis brimblecombei em Eucalyptus spp., em Petrolina, PE. Pesquisa Florestal Brasileira, Colombo, v. 30, p. 253-255, 2010. DOI: http://dx.doi.org/10.4336/2010.pfb.30.63.253

BRENNAN E. B.; GILL R. J.; HRUSA G. F.; WEINBAUM, S. A. First record of Glycaspis brimblecombei (Moore) (Homoptera: Psyllidae) in North America: initial observations and predator associations of a potentially serious new pest of Eucalyptus in California. Pan-Pacific Entomologist, San Francisco, v. 75, p. 55-57, 1999.

BRENNAN, E. B.; HRUSA, F.; WEINBAUM, S. A.; LEVISON JUNIOR, W. Resistance of eucalyptus species Glycaspis brimblecombei (Homoptera: Psyllidae) in the San Francisco Bay area. Pan Pacific Entomologist, San Francisco, v. 77, p. 249-253, 2001.

BRENNAN, E. B.; WEINBAUM, S. A. Effect of epicuticular wax on adhesion of psyllids to glaucous juvenile and glossy adult leaves of Eucalyptus globulus Labillardière. Australian Journal of Entomology, Canberra, v. 40, p. 270-277, 2001a.

BRENNAN, E. B.; WEINBAUM, S. A. Stylet penetration and survival of three psyllid species on adult leaves and 'waxy' and 'de-waxed' juvenile leaves of Eucalyptusglobulus. Entomologia Experimentalis et Applicata, Dordrecht, v. 100, p. 355-363, 2001 b.

BROOKER, M. I. H. A new classification of the genus Eucalyptus L'Hér. (Myrtaceae). Australian Systematic Botany, Melbourne, v. 13, p. 79-148, 2000.

BURCKHARDT, D. Psylloid pests of temperate and subtropical crop and ornamental plants (Hemiptera, Psylloidea): a review. Trends in Agricultural Sciences, Entomology, v. 2, p. 173-186, 1994.

BURCKHARDT, D.; LOZADA, P. W.; DIAZ, B. W. First record of the red gum lerp psyllid Glycaspis brimblecombei (Hemiptera: Psylloidea) from Peru. Bulletin de la Société entomologique Suisse, Zürich, v. 81, p. 83-85, 2008.

BURCKHARDT D.; OUVRARD D. A revised classification of the jumping plant-lice (Hemiptera: Psylloidea). Zootaxa, Auckland, v. 3509 , p. 1-34, 2012.

BURCKHARDT D.; QUEIROZ D. L. Commented checklist of the jumping plant-lice (Hemiptera: Psylloidea) from Brazil. Zootaxa, Auckland, v. 3571, p. 26-48. 2012.
CENTRE FOR PLANT BIODIVERSITY RESEARCH. Eucalyptus camaldulensis var. camaldulensis. In: EUCLID: eucalypts of Australia. 3rd ed. Canberra, 2006. Disponível em: $<$ http://www. anbg.gov.au/cpbr/cd-keys/euclid3/euclidsample/html/Eucalyptus camaldulensis_var._camaldulensis.htm>. Acesso em: 22 fev. 2013.

FERREIRA, R. de A.; BLAZIZA, A. A. B.; ANZOLIN, M. G.; FIRMINO-WINCKLER, D. C. Flutuação populacional do psilídeode-concha Glycaspis brimblecombei Moore (Hemiptera:Psyllidae) em Eucalyptus spp. no município de Garça. Revista Científica Eletronica de Engenharia Florestal, v. 14, p. 14, 2009.

FIRMINO-WINCKLER D. C.; WILCKEN, C. F.; OLIVEIRA, N. C.; MATOS, C. A. O. Biologia do psilídeo-de-concha Glycaspis brimblecombei Moore (Hemiptera, Psyllidae) em Eucalyptus spp. Revista Brasileira de Entomologia, São Paulo, v. 53, p. 144-146, 2009.

FLORESTAS plantadas respondem por $90 \%$ de toda a madeira produzida no país. Informativo ABRAF, Brasília, DF, ano 8, n. 375, p. 1, dez. 2013. Disponível em: <http://www.abraflor.org.br/ informativo/abraf375.html $>$. Acesso em: 30 jan. 2014.

HIDALGO REYS, F. O. Evaluación de la preferencia de Glycaspis brimblecombei Moore por diversos hospedadores del género Eucalyptus L'Herit. en la región metropolitana, Chile. Universidad de Chile, 2005. 40 f. Memoria (Profesional de Ingeniero Forestal) - Universidad de Chile. Santiago.

HODKINSON, I. D. Life cycle variation and adaptation in jumping plant lice (Insecta: Hemiptera: Psylloidea): a global synthesis. Journal of Natural History, London, v. 43, n. 1-2, p. 65-179, 2009.

HOLLIS, D. Australian Psylloidea: jumping plantlice and lerp insects. Canberra: Australian Biological Resorces Study, 2004. 232 p.

MELLIDO FILHO, A. Plano de manejo de rendimento sustentado da Fazenda Santa Cecília, Companhia Mineira de Metais. Belo Horizonte: Serviços Técnicos Ambientais, 1994. 28 p.

MOORE K. M. The Glycaspis spp. (Homoptera, Psyllidae) associated with Eucalyptus camaldulensis. Proceedings of the Linnean Society of New South Wales, Sydney, v. 99, p. 121-128, 1975.

MORA, A. L.; GARCIA, C. H. A cultura do eucalipto no Brasil. São Paulo: Sociedade Brasileira de Silvicultura, 2000. 112 p.

PAINE, T. D.; DAHLSTEN, D. L.; MILLAR, J. G.; HODDLE, M. S.; HANKS, L. M. UC scientists apply IPM techniques to new eucalyptus pest. California Agriculture, Berkeley, v. 54, p 8-13, 2000.

PEREIRA, J. M.; BALDIN, E. L. L.; SOLIMAN, E. P.; WILCKEN, C. F. Attractiveness and oviposition preference of Glycaspis brimblecombei Moore (Hemiptera: Psyllidae) in Eucalyptus spp. Phytoparasitica, Bet Dagan, v. 41, p. 117-124, 2013.

PERIS-FELIPO F. J.; MANCUSI G.; TURRISI, G. F.; JIMÉNEZPEYDRÓ, R. New chronological and biological data of the Red Gum Lerp Psyllid, Glycaspis brimblecombei Moore, 1964 in Italy (Hemiptera, Psyllidae). Biodiversity Journal, Palermo, v. 2, p. 13-17. 2011. 
PRYOR, L. D.; JOHNSON, L. A. A classification of the eucalyptus. Canberra: Australian National University, 1971. 192 p.

QUEIROZ, D. L.; BURCKHARDT, D.; MAJER, J. Integrated pest management of eucalypt Psyllids (Insecta, Hemiptera, Psylloidea). In: LARRAMENDY, M. L.; SOLONESKI, S. (Org.). Integrated pest management and pest control: current and future tactics. Rijeka: InTech, 2012. v. 1. p. 385-412. E-book.

QUEIROZ, D. L.; MAJER, J.; BURCKHARDT, D.; ZANETTI, R.; FERNANDEZ, J. I. R.; QUEIROZ, E. C. de; GARRASTAZU, M.; FERNANDES, B. V.; ANJOS, N. dos. Predicting the geographical distribution of Glycaspis brimblecombei (Hemiptera:Psylloidea) in Brazil. Australian Journal of Entomology, Canberra, v. 52, n. 1, p. 20-30, 2013.

SANTANA, D. L. Q.; ANJOS, N. Resistência de Eucalyptus spp. à Atta sexdens rubropilosa e Atta laevigata (Hymenoptera: Formicidae). Revista Árvore, Viçosa, MG, v. 13, n. 20, p. 174$181,1989$.

SANTANA, D. L. Q.; BELlote, A. F. J.; DEDECEK, R. A. Ctenarytaina spatulata, Taylor: água no solo, nutrientes minerais e suas interações com a seca dos ponteiros de eucalipto. Boletim de Pesquisa Florestal, Colombo, v. 46, p. 57-67, 2003a.

SANTANA, D. L. Q.; BURCKHARDT, D. Introduced eucalyptus psyllids in Brazil. Journal of Forest Research, v. 12, p. 337-344, 2007.
SANTANA, D. L. Q.; COUTO, E. L. Resistência intra-específica de eucaliptos a formigas cortadeiras. Boletim de Pesquisa Florestal, Colombo, n. 20, p.13-21, jun. 1990.

SANTANA, D. L. Q.; MENEZES, A. O.; SILVA, H. D.; BELLOTE, A. F.J .; FAVARO, R. M. O psilídeo-de-concha (Glycaspis brimblecombei) em eucalipto. Colombo: Embrapa Florestas, 2003b. 3 p. (Embrapa Florestas. Comunicado técnico, 105).

SANTANA, D. L. Q.; ZANOL, K. M. R.; OLIVEIRA, E. B.; ANJOS, N.; MAJER, J. Feeding and oviposition preferences of Ctenarytaina spatulata Taylor (Hemiptera: Psyllidae) on Eucalyptus spp. and other Myrtaceae growing in Brazil. Revista Brasileira de Entomologia, São Paulo, v. 54, p. 149-153, 2010.

WHITE, T. C. R. An index to measure weather-induced stress associated whit outbreaks of psyllids in Australia. Ecology, Tempe, v. 50, p. 905-9, 1969.

WILCKEN, C. F.; COUTO, E. B.; ORLATO, C.; FERREIRA FILHO, P. J.; FIRMINO, D. C. Ocorrência do psilídeo-de-concha Glycaspis brimblecombei em florestas de eucalipto no Brasil. Circular Técnica IPEF, Piracicaba, n. 201, p. 1-11. 2003.

ZANUNCIO, J. C. (Coord.). Manual de pragas em florestas: Lepidoptera desfolhadores de eucalipto: Biologia, ecologia e controle. Viçosa, MG: UFV/SIF, 1993. v. 1. 140 p. (Manual de pragas em florestas, 1). 
